\title{
TO STUDY THE THERAPEUTIC MANAGEMENT, DRUG RELATED PROBLEMS AND CONCOMITANT USE OF DRUGSIN PATIENTS WITH CANCER
}

\author{
PRAMILA P. ${ }^{1}$, ANJU ABRAHAM ${ }^{1}$, SUNITA PAWAR ${ }^{2}$, VIBHA BAFNA ${ }^{3}$, MONIKA S BANSAL ${ }^{1 *}$ \\ 1,2Department of Clinical Pharmacy, Poona College of Pharmacy, Bharati Vidyapeeth Deemed University, Pune, Pharm. D Interns, \\ ${ }^{3}$ Department, Bharati Hospital and Research Centre, Pune \\ Email: sunita70pawar@gmail.com
}

Received: 28 Feb 2017 Revised and Accepted: 29 Apr 2017

\section{ABSTRACT}

Objective: To study the prescribing patterns of chemotherapeutic drugs, concomitant drugs and to determine the drug-related problems in cancer patients.

Methods: A prospective and retrospective observational study was conducted over a period of 6 mo in a tertiary care teaching hospital, Pune after ethical approval and informed consent. Patients were then interviewed for patient information like demographics, treatment, and associated drug related problems using specially designed proforma and then required data was introduced in Microsoft excel spreadsheets.

Results: Out of 60 patients 50 were enrolled in this study during which 9 different sites of cancer were examined. The maximum number of patients with cancer resides in the age group of 51-60 y (32\%) and more common in females in 27 (54\%). The most prevalent risk factor and co-morbidity encountered were tobacco chewing $13(26 \%)$ and hypertension $8(16 \%)$, respectively. On the further evaluation of data, the findings suggested that the majority of patients were prescribed with an alkylating group of anti-neoplastic agents, paclitaxel+platinum-based compound regimen, and the drug paclitaxel. On screening, 167 drug interactions were observed, of these most of the interactions were in the moderate category. The most common organ system affected was gastrointestinal system 135 (30.80\%) whereas the prevalent toxicity was hyperuricemia. Polypharmacy was not observed, whereas antacid (ranitidine) was frequently prescribed during hospitalisation and discharge.

Conclusion: This study has highlighted certain facts and drawbacks in medication-related care which can be addressed by conducting future studies in cancer care in order to provide patient-specific outcomes.

Keywords: Cancer, Cytotoxics, Chemotherapeutic drugs, Concomitant drugs, Drug-related problems

(C) 2017 The Authors. Published by Innovare Academic Sciences Pvt Ltd. This is an open access article under the CC BY license (http://creativecommons.org/licenses/by/4.0/) DOI: http://dx.doi.org/10.22159/ijpps.2017v9i6.18207

\section{INTRODUCTION}

Cancer has been a vital public health problem with over 800,000 new cases occurring per annum in India. It is estimated that there are nearly 2.5 million cases within the country with nearly 400,000 deaths occurring because of cancer [1]. India holds the lowest 5-year survival for most cancer sites due to an underdeveloped and fragmented health care system [2]. A person's risk of developing cancer is dependent on many factors, including age, genetics, and exposure to risk factors (including some potentially avoidable lifestyle factors). Cancer risk factors are overall similar worldwide. Smoking, insufficient physical activity, alcohol, diet, overweight and obesity, sun exposure and infections account for a high proportion of cancers worldwide [3]. All types of cancers have been reported in the Indian population including cancers of skin, lung, breast, rectum, stomach, prostate, liver, cervix, oesophagus, bladder, blood, mouth [4]. Increasing trends of cancer prevalence seen nowadays have become an important agenda of the health sector of every country signifying a continuous need for better cancer therapies.

Different modalities of treatment include radiation, surgery, chemotherapy, hormonal therapy, immunotherapy, biologic therapy, and cryosurgery. The treatment has been done more tumors specific and less toxic with the help of novel cancer targeted therapies. Depend on the type and stage of cancer; patients obtain a unique tumor treatment protocol. Chemotherapy is used as a part of a multimodal approach to the preliminary treatment of different types of tumors. Various drugs used are gemcitabine, gefitinib, azacitidine, paclitaxel, carboplatin, docetaxel, cyclophosphamide, 5-flurouracil, methotrexate, daunorubicin etc. Superior clinical outcomes may be produced by a dose-dense regimen of combination chemotherapy. Nowadays most treatments are premeditated specifically for each individual [5]. Frequently observed, supportive care drugs are gastrointestinal drugs, corticosteroids, anti-histaminic, analgesics, antibiotics, nutritional, iron and vitamin supplements [6].
The major obstacle in the treatment of cancer is resistant to chemotherapy. Drug resistance either acquired or intrinsic often prevents tumor cells from undergoing sufficient levels of programmed cell death or apoptosis leading to the survival of cancer cells and treatment failure [7]. Numerous complexes with biological activity act as anticancer agents have been investigated, however many of them are not suitable for therapeutic use owing to their toxic, carcinogenic and mutagenic properties. The use of chemotherapeutic drugs in cancer therapy involves the risk of lifethreatening host toxicity. The search continues to develop the drugs which selectively act on tumor cells [8].

Chemotherapeutic drugs have a narrow therapeutic index due to which the rapidly dividing tissue gets adversely affected. These effects range from mild nausea to myelosuppression [9]. Common toxicities encountered are hematological, gastrointestinal, nervous system, cardiac and pulmonary toxicities [10]. Depending upon the complexity of the illness, cancer patient's needs multiple drugs for management of their co-morbid conditions putting them at high risk for complications caused by drug-drug interactions and associated polypharmacy [11].

Pharmacists can play a key role in the management of dose modifications, helping patients gain access to treatment influencing them to remain adherent, identifying treatment related dose toxicities and educating about potential conflicts in the treatment regimen. With this background, this study was aimed to describe the prescribing pattern of anticancer drugs along with adjuvant drugs, the pattern of adverse drug reaction, toxicities and polypharmacy encountered during chemotherapy.

\section{MATERIALS AND METHODS}

An observational, prospective and retrospective record based study was carried out in General medicine, Surgery and Paediatric department of tertiary care teaching hospital, Pune, over a period of 6 mo during the year September 2015 to February 2016, after 
receiving the approval from the institutional ethics committee with an ethics vide letter number (BVDU/MC/95).

Out of 60 patients, data of 50 patients were enrolled in the study. All patient related information were collected from the history file and medical records after taking written informed consent and interviewing patient and their caregivers. The subjects who had willingly participated were enrolled on the basis of inclusion and exclusion criteria.

\section{Inclusion criteria}

Patients of either sex or gender diagnosed with cancer three years before and newly diagnosed cases up to the third stage of cancer, undergoing chemotherapy, radiation, and surgery. Also, patients with co-morbid conditions along with cancer were enrolled in the study.

\section{Exclusion criteria}

Patients with more than three co-morbid conditions as well as those undergoing biotherapy and immunotherapy were excluded.

\section{Data collection}

The data collection proforma was developed and used which includes patient as well as medication-related information. The drug-related problems such as possible drug interactions were analysed by using Micromedex drug interaction software, adverse drug reactions as per reference to WHO criteria and toxicities as per pharmacology handbook. Further, this data was correlated with persistent complaints on each follow-up to observe the occurrence of the event.

\section{Statistical analysis}

Data collected was introduced into Microsoft Excel 2007 and analysed for descriptive statistics, frequency percentage and presented in the tabular and graphical form.

\section{RESULTS}

In a study period of $6 \mathrm{mo}$, a total number of patients screened for malignancy were 60 patients in which 9 different sites of cancers (Non-Hodgkin's lymphoma, breast, rectal, oral, stomach, esophageal, ovarian, leukemia, and prostate cancers) were observed. Based on inclusion and exclusion criteria stated in the protocol 50 patients were enrolled in the study and their medical records were reviewed prospectively and retrospectively, whereas 3 patients were excluded as they did not meet the inclusion criteria and 7 patients left followup from Bharati hospital.

\section{Demographics}

Among the 50 patients enrolled, 23 were males and 27 were females. Gender and age group analysis of diagnosed cases of cancer revealed that incidence of cancer increased noticeably after an age of $30 \mathrm{y}$. It was further seen that cancer was most dominant in the females. The prevalent pattern showed maximum patients within the age interval of 51-60 y (32\%) and minimum in $11-20$ y (4\%). The social habits and the past medical history of the study population were observed and it was found that most of the patients were having the habit of tobacco chewing followed by mishri application whereas hypertension and diabetes mellitus to be a prominent co-morbid condition (table 1).

Table 1: Demographic characteristics of cancer patients

\begin{tabular}{|c|c|c|c|}
\hline \multicolumn{2}{|l|}{ Characteristics } & \multirow[t]{2}{*}{ Number of patients $(n=50)$} & \multirow[t]{2}{*}{ Percentage (\%) } \\
\hline Gender & & & \\
\hline & Male & 23 & 46 \\
\hline & Female & 27 & 54 \\
\hline \multicolumn{4}{|l|}{ Age (Y) } \\
\hline & $\leq 10$ & 4 & 8 \\
\hline & $11-20$ & 2 & 4 \\
\hline & $21-30$ & 5 & 10 \\
\hline & $31-40$ & 7 & 14 \\
\hline & $41-50$ & 7 & 14 \\
\hline & $51-60$ & 16 & 32 \\
\hline & $61-70$ & 4 & 8 \\
\hline & $\geq 70$ & 5 & 10 \\
\hline \multicolumn{4}{|l|}{ Social habits } \\
\hline & Tobacco chewer & 13 & 26 \\
\hline & Alcohol consumer & 7 & 14 \\
\hline & Mishri applicant & 5 & 10 \\
\hline & Smoker & 2 & 4 \\
\hline \multicolumn{4}{|c|}{ Co-morbid condition } \\
\hline & Hypertension & 8 & 16 \\
\hline & Diabetes mellitus & 5 & 10 \\
\hline & Other (Bronchial asthma, Tuberculosis) & 8 & 16 \\
\hline
\end{tabular}

\section{Drug usage pattern}

In the present study, cancer treatment modality most widely used was chemotherapy alone in $50(100 \%)$ patients which are quite significant followed by combined modality therapy using two or more modalities such as $36(72 \%)$ underwent chemotherapy and surgery whereas 15 (30\%) underwent chemotherapy, surgery, radiation.

From the data collected it was observed that most frequently prescribed combination chemotherapeutic regimen was paclitaxel+platinum-based compounds accounting for $28 \%$ of the patients. For the treatment pattern of cancer and other patient information, the patient history file and other medical records were verified. Prescribing pattern of chemotherapeutic classes of cytotoxic drugs in study population showed that alkylating agents were prominently prescribed in clinical settings and it accounted nearly $43(86 \%)$ of the study population followed by the class antimetabolites 34 (68\%), plant alkaloids 30 (60\%) and antitumor antibiotics $16(32 \%)$.

Further analysis suggested that commonly prescribed chemotherapeutic agent was paclitaxel 17 (34\%) followed by 5-fluorouracil $16(32 \%)$, cisplatin $13(26 \%)$ and doxorubicin 9 (18\%). Among the utilization pattern of adjuvant drugs for the management of adverse effects of anticancer drugs ranitidine (antacids) was frequently prescribed followed by dexamethasone (corticosteroids), granisetron (antiemetic) and promethazine (antihistaminic) during hospitalization whereas ranitidine (antacids) 76\% followed by lorazepam (anxiolytic) $70 \%$, granisetron, and prochlorperazine (anti-emetic) 68\%, Dulcolax (laxative) 64\% during discharge (table 2). 
Table 2: Drug use pattern

\begin{tabular}{|c|c|c|c|}
\hline \multicolumn{4}{|c|}{ Chemotherapeutic drug usage pattern } \\
\hline Medication class & Chemotherapeutic Drugs & Number of patients $(n=50)$ & Percentage (\%) \\
\hline \multirow[t]{5}{*}{ Alkylating agents } & & 43 & 86 \\
\hline & Cisplatin & 13 & 26 \\
\hline & Carboplatin & 12 & 24 \\
\hline & Cyclophosphamide & 9 & 18 \\
\hline & Oxaliplatin & 8 & 16 \\
\hline \multirow[t]{5}{*}{ Antimetabolites } & & 34 & 68 \\
\hline & 5-Fluorouracil & 16 & 32 \\
\hline & Methotrexate & 6 & 12 \\
\hline & Cytarabine & 4 & 8 \\
\hline & Gemcitabine & 4 & 8 \\
\hline \multirow[t]{5}{*}{ Plant alkaloids } & & 30 & 60 \\
\hline & Paclitaxel & 17 & 34 \\
\hline & Vincristine & 9 & 18 \\
\hline & Docetaxel & 3 & 6 \\
\hline & Etoposide & 1 & 2 \\
\hline \multirow[t]{4}{*}{ Antitumor antibiotics } & & 16 & 32 \\
\hline & Doxorubicin & 9 & 18 \\
\hline & Daunorubicin & 6 & 12 \\
\hline & Bleomycin & 1 & 2 \\
\hline \multirow[t]{2}{*}{ Enzymes } & & 5 & 10 \\
\hline & L-asparaginase & 5 & 10 \\
\hline \multirow[t]{3}{*}{ Glucocorticosteroids } & & 4 & 8 \\
\hline & Prednisolone & 2 & 4 \\
\hline & Prednisone & 2 & 4 \\
\hline \multirow[t]{2}{*}{ Monoclonal antibodies } & & 1 & 2 \\
\hline & Rituximab & 1 & 2 \\
\hline \multirow[t]{2}{*}{ Hormonal agents } & & 1 & 2 \\
\hline & Megestrol & 1 & 2 \\
\hline \multirow[t]{2}{*}{ Miscellaneous agents } & & 1 & 2 \\
\hline & Hydroxyurea & 1 & 2 \\
\hline \multicolumn{4}{|l|}{ Concomitant drug usage pattern } \\
\hline Indication & Drug & Number of patients $(n=50)$ & No. of Patients (\%) \\
\hline Antacids & Ranitidine & 36 & 72 \\
\hline Corticosteroids & Dexamethasone & 32 & 64 \\
\hline Nausea and Vomiting & Granisetron & 27 & 54 \\
\hline Anti-histaminic & Promethazine & 26 & 52 \\
\hline Vitamins & Becosule & 15 & 30 \\
\hline Analgesic and Antipyretic & Diclofenac & 11 & 22 \\
\hline Antibiotics & Ceftriaxone & 12 & 24 \\
\hline Anti-diarrheal & Vibact & 8 & 16 \\
\hline Antifungal & Fluconazole & 6 & 12 \\
\hline Folic acid supplement & Orofer XT & 6 & 12 \\
\hline Mouth and Throat preparations & Betadine gargles & 6 & 12 \\
\hline Anxiolytic & Lorazepam & 6 & 12 \\
\hline Hematopoietic agents & Filgrastim & 6 & 12 \\
\hline Laxative & Duphalac & 5 & 10 \\
\hline
\end{tabular}

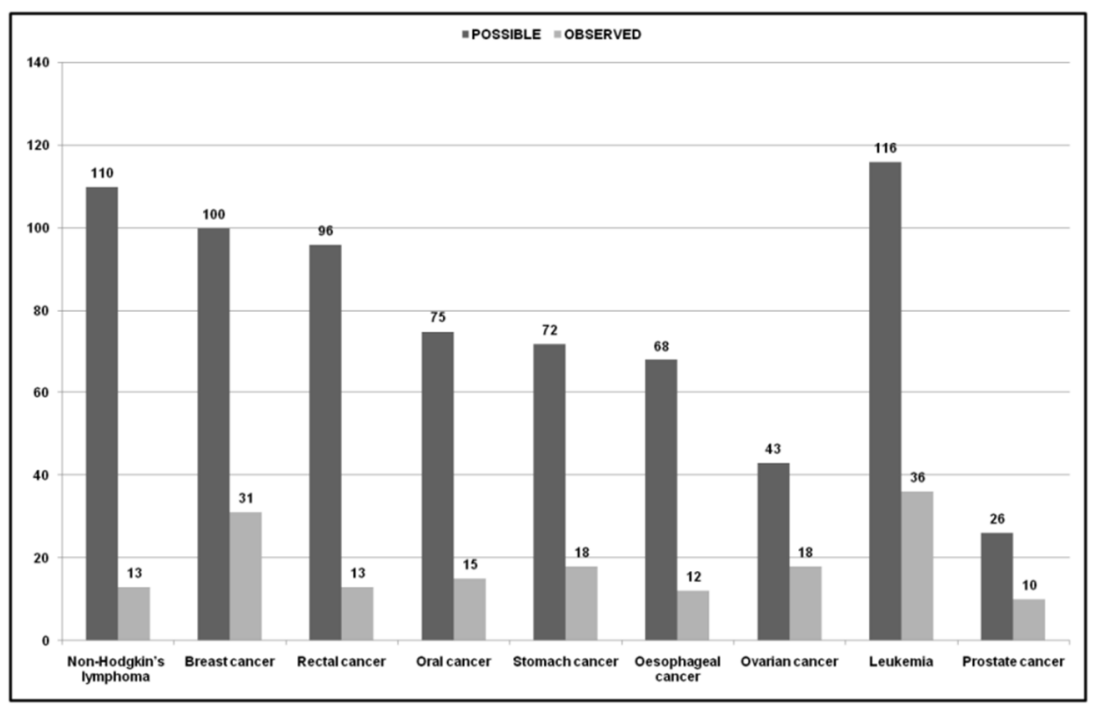

Fig. 1: Drug interaction observed in each cancer type of study population 


\section{Drug-related problems}

\section{Drug interaction}

Drug interaction screening tool identified a total of 706 potential drug interactions, of which 167 were clinically relevant and required clinical interventions. Based on the severity, 34 interactions were major, 131 were moderate and 2 were minor. The further evaluation of drug interaction data in each cancer is observed as (fig. 1).

The chemotherapeutic drug that was identified most frequently as having the potential for interaction was methotrexate followed by cisplatin, doxorubicin, and cyclophosphamide while among concomitant drugs prochlorperazine followed by lorazepam, metoclopramide and promethazine had the potential to interact with the majority of drugs prescribed (table 3 ).

\section{Adverse drug reactions}

Out of 448 total adverse drug reactions encountered, the present study showed that in both males and females $(n=50)$, the most affected organ system was gastrointestinal system with 135 adverse drug reactions $(30 \%)$ followed by general body disorders with 75 adverse drug reactions (17\%), blood disorders with $68(15 \%)$, neurological disorders with $40(9 \%)$, liver disorders with $38(8 \%)$, respiratory system with 22 (5\%), skin disorders with $20(4 \%)$, cardiovascular disorders with $18(4.01 \%)$ and urinary system disorders with $17(3.7 \%)$. Further observation of the most prominent adverse effect as per organ-system is shown in (table 4).

Table 3: Potential drugs involved in drug interaction in study population

\begin{tabular}{ll}
\hline Chemotherapeutic agents & No. of interaction \\
\hline Drugs & 14 \\
Methotrexate & 11 \\
Cisplatin & 9 \\
Doxorubicin & 6 \\
Cyclophosphamide & No. of interaction \\
Concomitant drugs & 40 \\
Drugs & 24 \\
Prochlorperazine & 23 \\
Lorazepam & 23 \\
Metoclopramide & \\
Promethazine & \\
\hline
\end{tabular}

Table 4: Prominent adverse effects observed in study population

\begin{tabular}{lll}
\hline Adverse drug reactions & & No. of patients (\%) (n=50) \\
\hline Organ system & Most prominent adverse effect & $38 \%$ each \\
\hline Gastrointestinal system & Nausea and Vomiting & $28 \%$ \\
General body disorders & Fever & $70 \%$ \\
Blood disorders & Anaemia & $26 \%$ \\
Neurological disorders & Dizziness & $48 \%$ \\
Liver disorders & Clinical biochemistry fluctuations & $24 \%$ \\
Respiratory system disorders & Dyspnoea & $20 \%$ \\
Skin and appendages & Skin rash/Itching/Pigmentation & $12 \%$ \\
Cardiovascular disorders & Blood pressure fluctuations & $8 \%$ \\
Urinary system disorders & Polyuria & \\
\hline
\end{tabular}

Table 5: Prominent toxicities observed in study population

\begin{tabular}{lll}
\hline Toxicity & & \\
\hline A. General Toxicities & Nyperuricaemia & 5 \\
& Bone Marrow Suppression & 2 \\
& Immunosuppression & 1 \\
& Gastrointestinal tract toxicity & 1 \\
& Skin And Hair & 1 \\
& Carcinogenicity & 1 \\
& Infertility & 1 \\
& Renal Dysfunction & 1 \\
& & \\
& $\bullet \quad$ Cardiotoxicity induced by doxorubicin, paclitaxel, and daunorubicin. \\
& $\bullet \quad$ Cyclophosphamide-induced hemorrhagic cystitis & 5 \\
& $\bullet \quad$ Cisplatin-induced nephrotoxicity & 1 \\
& $\bullet \quad$ Neuropathy induced by vincristine and paclitaxel \\
\hline
\end{tabular}

\section{Toxicity}

From the data collected, it was observed that most commonly observed toxicity from general category were hyperuricemia followed by bone marrow suppression, immunosuppression, carcinogenicity, infertility, renal dysfunction, gastrointestinal (nausea/vomiting), and skin/hair was encountered. When observed in a specific category cardio toxicity was prominent followed by hemorrhagic cystitis, nephrotoxicity, and neuropathy. The further classification based on general and specific toxicities are observed and mentioned in table 5.

\section{DISCUSSION}

Trends in cancer types and their incidence are growing day by day in a developing country like India, which is showing a major impact on 
the socio-economic status of people. During a study of $6 \mathrm{mo}$, we included 50 patients admitted in General Medicine, Surgery and Paediatric department of a tertiary care teaching hospital, Pune. On analyzing the distribution pattern of cancer patients according to gender, the data represented that cancer was more prevalent in females $(54 \%)$ than males $(46 \%)$ in the age between $1-80 \mathrm{y}$. This data was found to be consistent with the observation of female (52.14\%) and male (47.85\%) as reported by Kulkarni et al., [13]. The greater prevalence of cancer in females can be due to the involvement of their reproductive system which is more susceptible such as cervix, ovary, and breast and occupies the major portion of all other forms of cancer. Gender differences in susceptibility to a disease are a very useful piece of information that can be used to develop a causal hypothesis for the disease, to define subgroups at higher risk and contribute important clues for etiology of cancers. Age wise distribution of the patients showed that there was a higher incidence of cancer in the age group of 51-60 y (32\%). Similar findings were observed as a higher incidence of cancer in the age group of (50-60) years and (46-60) years in the studies carried out by Pentareddy et al., and Sneha et al., respectively [12, 17]. Cancer prevalence trend appears to increase with age. The accumulation of age-associated changes in a biochemical process that help control genes may be the cause of some of the increased risk of cancer in older people, according to National Institute of Health study.

In the current study, it was observed that tobacco chewing habit was more prominent in cancer patients $(26 \%)$ followed by alcohol drinking (14\%). This observation is in agreement with the study conducted by Sloan et al. which reports tobacco chewing as the most significant factor for cancer and across the board for chronic diseases. Tobacco chewing had 2.5 times higher occurrence of potentially malignant diseases. It was found that there was a synergistic effect of smoking, tobacco chewing and alcohol drinking towards the development of cancer implying the fact that the presence of one risk factor enhanced the effects of subsequent risk factor [18]. The highest percentage of hypertension (16\%) was identified as a co-morbid condition among cancer patients. This was consistent with the findings of Piccirillo et al., [19].

For the treatment of cancer, various modalities such as chemotherapy, radiation therapy, immunotherapy and monoclonal antibody therapy are used. In the present study use of chemotherapy as a management modality was observed in $100 \%$ of patients followed by chemotherapy+surgery (72\%) and chemotherapy+surgery+radiation $(30 \%)$. A study was done by Nazmul et al. found that maximum cancer patients took chemotherapy (40.4\%) and $23.4 \%$ patients took both surgery and chemotherapy [20]. Choice of therapy depends upon the location and grade of the tumor. According to a study done by Kiebert et al., six out of seven factors were found to be of considerable importance when treatment choice for cancer patients was made. The seven factors were the age at the time of the decision, having a partner, having children, inability to work due to side-effects, the nature of the side-effects, disease-related life expectancy and baseline quality of life influenced the selection [21]. In our study, Paclitaxel was commonly prescribed chemotherapeutic agent (34\%) followed closely by 5fluorouracil $(32 \%)$. This was quite similar to the results reported by Kulkarni et al., showing carboplatin followed by paclitaxel [13]. Among the different classes of cytotoxic drugs observed, alkylating agents was the most prescribed class followed by antimetabolites, antitumor antibiotics, and plant alkaloids. This result was supported by the study conducted by Khan et al., [14]. Anticancer drugs were mostly prescribed in combination in the present study (90\%). This finding is significant with the existing utilization pattern of anticancer drugs reported in studies done by Pentareddy et al., (81.21\%) and Dave et al., $(94.62 \%)$, respectively $[12,15]$. The fundamental principle of combination chemotherapy is that different drugs act through cytotoxic mechanisms. Among the combination chemotherapeutic regimens, paclitaxel and platinum-based combinations were mostly prescribed. Comparable results were reported by Ramalakshmi S et al., [6]. Judicious choice of alkylating agents given in sequential or concurrent combination may be a rational treatment strategy with potential applications in the clinic.

The commonly used adjuvant drugs in our study were ranitidine $(72 \%)$ and granisetron (54\%) followed by dexamethasone $(64 \%)$ and lorazepam (12\%). Comparably according to Vrabel nausea and vomiting are the most distressing side effects of cancer chemotherapy and the result reported in the study revealed that ondansetron and granisetron have comparable antiemetic efficacy in reducing or eliminating chemotherapy-induced nausea and vomiting [16]. In the present study, granisetron (54\%) was more commonly prescribed followed by ondansetron (40\%) and prochlorperazine (16\%) for nausea and vomiting.

In the present study drug-drug interactions were observed to be 167 out of which $20 \%$ was major, $78 \%$ was moderate and $1 \%$ was minor, this does not differs greatly from the study carried out by Kannan et al.,which projects reporting of 213 interactions out of which $9 \%$ was major, 56\% was moderate and 33\% minor respectively [22]. Further in the present study, among chemotherapeutic drugs, it was found that methotrexate was the most potentially interacting drug followed by cisplatin and doxorubicin. Similar findings were observed in the study carried out by Voll et al., which showed the anti-cancer drug most involved in the drug-drug interactions methotrexate [23]. Further in present study, amongst the concomitant drugs prochlorperazine (anti-emetic) followed by lorazepam (anxiolytic), metoclopramide (antiemetic and gastrointestinal regulator) and promethazine (anti-vertigo) showed more potential to interact with other prescribed drugs which was incompatible with the results reporting antiretroviral drugs followed by proton pump inhibitors and antibiotics to be more potential as interacting drugs in the study conducted by Voll et al., [23].

This study identified 448 adverse drug reactions of which about 135 adverse drug reactions were more prominent in the gastrointestinal system $30 \%$, followed by general body disorders $17 \%$ and blood disorders $15 \%$ this does not differs greatly to the study done by Prasad et al., which reports that the most common adverse drug reactions found are nausea and vomiting that is related to gastrointestinal system followed by neutropenia and anemia which belongs to blood disorders [24]. Also on further individualised specification as per adverse effect the present study depicts gastrointestinal system to be affected the most which include constipation, nausea, vomiting followed by general body disorders like fatigue which is comparable to the result depicting the most prominent adverse effects were constipation, nausea, vomiting, fatigue, alopecia and drowsiness in the study carried out by Lau et al.,[25].

In the current study, hyperuricaemia and cardiotoxicity were the most commonly observed toxicity from general category whereas in specific category cardiotoxicity in 5 patients out of 50 receiving doxorubicin, daunorubicin, paclitaxel was prominent which is similar to the study done by Alexander et al., which shows cardiotoxicity in 5 patients out of 55 receiving doxorubicin and in another study by Shek TW Luk IS et al., projects cardiotoxicity is induced by paclitaxel and anthracyclines [26, 27].

In the reference study done by Korc-Grodzicki et al., it is reported that nearly one-third of community-dwelling adults aged 65 or older are prominent to take more than 5 prescription medications and almost $20 \%$ take 10 or more [28]. The prevalence rates of polypharmacy and potentially inappropriate medications in older adults with newly diagnosed cancer were $80 \%$ and $41 \%$ respectively in the study conducted by Sokol et al., [29]. But in the current study polypharmacy parameter is not observed as the sample size of the study population was less and maximum numbers of patients were in the age group below $60 \mathrm{y}$ whereas, polypharmacy is most frequently observed in older adults aged greater than $65 \mathrm{y}$ and due to age-related multiple co-morbidities and frequent administration of Over-the-Counter medications.

\section{CONCLUSION}

This study provides insight into the following aspects of drug use, drug prescribing and drug-related problems. The prevalence of carcinoma was seen more in females than males, prominent in the age group (5160years) with hypertension and tobacco chewing habit to be significantly observed risk factor and social habit, respectively. The chemotherapeutic drugs have a narrow therapeutic index and the dosage needed to achieve a therapeutic response, many times leads to drug-related problems. Early detection of such drug-related problems 
helps in modifying the drug regimen or doses to reduce the adverse events. So, such studies are needed to assist the healthcare team in cognising the use of appropriate drug therapy, drug related problem with its management and development of alert guidelines and computer-based screening so that overall positive patient outcomes are achieved.

\section{AUTHORS CONTRIBUTION}

First author: Monika Bansal

Second author: Pramila Jadhav

Third author: Anju Abraham

Guide and corresponding Author: Sunita Pawar

Co-guide: Dr. Vibha Bafna

\section{Limitations}

The short duration of the study, limited sample size, and patients lost to follow-up due to certain conditions.

\section{CONFLICT OF INTERESTS}

\section{Declared none}

\section{REFERENCES}

1. Sanigaram V, Lingampally S, Boyana A, Vurumadla S. A prospective study on clinical evaluation, treatment pattern and adverse effects of anticancer drugs in various gynaecological cancer patients. Asian J Pharm Clin Res 2015;8:125-31.

2. Sankaranarayanan R. Cancer prevention and care in India: an unfinished agenda. Lancet Oncol 2014;15:554-5.

3. Saka S, Singh A, Sharma N. Potential anti-cancer superfoods: a minireview. Int J Curr Pharm Res 2016;8:19-21.

4. Ali I, Waseem W, Saleem K. Cancer scenario in India with future perspectives. Cancer Ther 2011;8:56-70.

5. Gupta M, Dahiya J, Marwaha RK, Dureja H. Therapies in cancer treatment: an overview. Int J Pharm Pharm Sci 2015;7:1-9.

6. Ramalakshmi S, Ramesh A, Sahini K, Babu K, Kousalya K, Saranya P. A study on prescribing trends of supportive care drugs used in cancer chemotherapy in a tertiary care teaching hospital. Indian J Pharm Practice 2013;6:36-9.

7. Patel S, Singh N, Kumar L. Anticancer role of antidiabetic drug metformin in ovarian cancer cells. Int J Cancer Ther Oncol 2016;4:1-8.

8. Ali M, Mahmoud A, Abdel-Halim A, Fyiad A. Anti-cancer effect of some prepared sulfated oligosaccharides on three different human cancer cell lines. Asian J Pharm Clin Res 2014;7 Suppl 1:168-76.

9. Kirthi C, Afzal A, Reddy M, Ali S, Yerramilli A, Sharma S. A study on the adverse effects of anticancer drugs in an oncology center of a tertiary care hospital. Int J Pharm Pharm Sci 2014;6, Suppl 2:580-3.

10. Arora S, Agarwal S, Singhal S. Anticancer activities of thiosemicarbazides/thiosemicarbazones: a review. Int J Pharm Pharm Sci 2014;6:34-41.

11. Kotlinska-Lemieszek A, Paulsen O, Kaasa S, Klepstad P. Polypharmacy in patients with advanced cancer and pain: a European cross-sectional study of 2282 patients. J Pain Symptom Manage 2014;48:1145-59.

12. Pentareddy MR, AVS Suresh, Shailendra D, Subbaratnam Y, Prasuna G, DTV Naresh, et al. Prescription patterns of anticancer drugs in a tertiary care hospital. J Evidence Based Med Healthcare 2015;2:3001-9.
13. Kulkarni MD, Hussaini S, Padwal SL, Khandelwal P, Doifode S, More $\mathrm{P}$, et al. Drug utilization review of anticancer drugs in cancer outpatient department of the Government Medical College, Aurangabad. Int J Basic Clin Pharmacol 2014;3:879-83.

14. Khan GM, Thapa RK, Adhikari DS, Rajbhandari M, Dwa P Shrestha S, et al. Evaluation of cancer prevalence and cytotoxic medication prescribing in the central region of Nepal. Kathmandu University J Sci Eng Technol 2013;9:189-99.

15. Dave D, Pillai A, Shah D, Agarwal S, Goel A. An analysis of utilization pattern of anticancer drugs in diagnosed cases of carcinoma in a tertiary care teaching hospital. Int J Basic Appl Med Sci 2014;4:251-9.

16. Vrabel M. Is ondansetron more effective than granisetron for chemotherapy-induced nausea and vomiting? A review of comparative trials. Clin J Oncol Nurs 2007;11:809-13.

17. Sneha G, Aparna S, BNVB Sri Lakshmi, Varma S, Naidu DSR. Adjunctive drug utilization pattern in the oncology department of a tertiary care hospital in South India. Indo Am J Pharm Res 2015;5:1297-303.

18. Sloan FA, Gelband H. Cancer control opportunities in low-and middle-income countries. Washington (DC): National Academies Press (US); 2007. p. 1-4.

19. Piccirillo J, Tierney R, Costas I, Grove L, Spitznagel EL Jr. Prognostic importance of comorbidity in a hospital-based cancer registry. JAMA 2004;291:2441-7.

20. Nazmul H, Mesbah U, Rafiquzzaman Md, Chowdhury S, Wahed B. Distribution of types of cancer and patterns of cancer treatment among the patients at various hospitals in Dhaka division, Bangladesh. Int Res J Pharm 2012;3:219-22.

21. Kiebert GM, Stiggelbout AM, Kievit J, Leer JW, Van de Velde CJ, de Haes HJ. Choices in oncology: factors that influence patients' treatment preference. Qual Life Res 1994;3:175-82.

22. Kannan G, Anitha R, Rani V, Thennarasu P, Alosh J, Vasantha J, et al. A study of drug-drug interactions in cancer patients of a south Indian tertiary care teaching hospital. J Postgrad Med 2011;57:206-10.

23. Voll ML, Yap KD, Terpstra WE, Crul M. Potential drug-drug interactions between anticancer agents and community pharmacy dispensed drugs. Pharm World Sci 2010;32:575-80.

24. Prasad A, Datta P, Bhattacharya J, Pattanayak C, Chauhan A, Panda P. Pattern of adverse drug reactions due to cancer chemotherapy in a tertiary care teaching hospital in Eastern India. J Pharmacovigilance 2013;1:1-4.

25. Lau PM, Stewart K, Dooley M. The ten most common adverse drug reactions (ADRs) in oncology patients: do they matter to you? Support Care Cancer 2004:12:626-33.

26. Alexander J, Dainiak N, Berger HJ, Goldman L, Johnstone D, Reduto L, et al. Serial assessment of doxorubicin cardiotoxicity with quantitative radionuclide angiocardiography. N Engl J Med 1979;300:278-83.

27. Shek TW Luk IS, Ma L, Cheung KL. Paclitaxel-induced cardiotoxicity. An ultrastructural study. Arch Pathol Lab Med 1996;120:89-91.

28. Korc-Grodzicki B, Boparai M, Lichtman S. Prescribing for older patients with cancer. Clin Adv Hematol Oncol 2014;12:309-18.

29. Sokol KC, Knudsen JF, Li MM. Polypharmacy in older oncology patients and the need for an interdisciplinary approach to sideeffect management. J Clin Pharm Ther 2007;32:169-75.

\section{How to cite this article}

- $\quad$ Pramila P, Anju Abraham, Sunita Pawar, Vibha Bafna. To study the therapeutic management, drug related problems and concomitant use of drugsin patients with cancer. Int J Pharm Pharm Sci 2017;9(6):139-144. 